\title{
Baryogenesis vs. proton stability in theories with extra dimensions
}

\author{
Antonio Masiero*, Marco Peloso ${ }^{\dagger}$, Lorenzo Sorbo ${ }^{\ddagger}$, and Rula Tabbash ${ }^{\S}$ \\ Scuola Internazionale Superiore di Studi Avanzati (S.I.S.S.A.), \\ Via Beirut 2-4, I-34014 Trieste, Italy \\ and \\ Istituto Nazionale di Fisica Nucleare, Sez. di Trieste \\ Via Valerio 2, I-34127 Trieste, Italy
}

(March 31, 2000)

\begin{abstract}
We propose a new scenario of baryogenesis in the context of theories with large extra dimensions. The baryon number is almost conserved at zero temperature by means of a localization mechanism recently analyzed by Arkani-Hamed and Schmaltz: leptons and quarks are located at two slightly displaced positions in the extra space, and this naturally suppresses the interactions which "convert" the latter in the former. We show that this is expected to be no longer true when finite temperature effects are taken into account. The whole scenario is first presented in its generality, without referring to the bulk geometry or to the specific mechanism which may generate the baryon asymmetry. As an example, we then focus on a baryogenesis model reminiscent of GUT baryogenesis. The Sakharov out of equilibrium condition is satisfied by assuming nonthermal production of the bosons that induce baryon number violation.
\end{abstract}

PACS: 98.80.Cq, 11.10.Kk

Preprint : SISSA 31/2000/EP

\section{INTRODUCTION}

Despite the great success of Quantum Field Theory, a consistent scenario where gravity is also included still lacks. The most promising framework that could help in this task is string theory, whose consistency requires additional dimensions beyond the standard $3+1$. This extra space is usually assumed to be compact, with a small compactification radius of order $M_{p}^{-1}$. However, it has been observed in ref. [1] that, having no test of gravity below the millimeter scale, we do not really need such a tiny compactification radius, provided the extra dimensions are accessible only to gravitational interactions. The Standard Model degrees of freedom must indeed be localized on a 3 dimensional wall whose inverse thickness does not exceed the scale of energy, of order $\mathrm{TeV}$, we currently probe in accelerator experiments.

The choice of such large compactification scale has the main goal of solving (or at least of weakening) the hierarchy problem. Denoting by $V_{n}$ the volume of the compact space - assumed in [1] to have a trivial metric - and by $M$ the fundamental scale of gravity, the observed Planck mass is obtained by the relation $M_{p}^{2}=M^{n+2} V_{n}$. Under the condition $n \geq 2, M$ can be safely assumed to be very close to the electroweak scale, without conflicting with either cosmological, astrophysical, or laboratory bounds.

Although considerably improving the standard situation, the above scenario retains however some degree of fine tuning, connected to the largeness of the quantity $V_{n} \cdot M^{n}$. A better result in this regard is provided by the more recent work [2], where, due to the presence of cosmological constants in the bulk and on two walls, the metric is nonfactorizable with an exponential scaling in the extra space. This fact allows the achievement of both a (phenomenologically) acceptable theory with just one extra dimension (that could be even infinite in extension [3]) and a more satisfactory solution to the hierarchy problem.

There are some other aspects common to all of these theories besides the ones discussed above. In particular, both proton stability and baryogenesis may be problematic in models with very low fundamental masses.

For what concerns proton stability in Grand Unified Theories, the standard way to achieve it is to increase the mass of the additional bosons up to about $10^{15}-10^{16} \mathrm{GeV}$. In the framework of theories with extra-dimensions,

\footnotetext{
*E-mail address: masiero@sissa.it

${ }^{\dagger}$ E-mail address: peloso@sissa.it

${ }^{\ddagger}$ E-mail address: sorbo@sissa.it

${ }^{\S}$ E-mail address: rula@sissa.it
} 
an interesting mechanism has been suggested in ref. 洵. 1 In this paper, a dynamical mechanism for the localization of fermions on the wall [8] is adopted: leptons and quarks are however localized at two slightly displaced positions in the extra space, and this naturally suppresses the interactions which "convert" the latter in the former.

However, the observed baryon asymmetry requires baryon number $(B)$ violating interactions to have been effective in the first stages of the evolution of the Universe. In this paper we thus wonder how this last requirement can be satisfied in a theory which adopts the idea of [ [- to ensure proton stability now and baryon production in the past. Our proposal is that thermal corrections, which are naturally relevant at early times, may modify the localization of quarks and leptons so to weaken the mechanism that suppresses the $B$ violating interactions. 2

The plan of the work is the following. In section 2 we review the mechanism used in ref. [4] to localize chiral fermions on a domain wall and to suppress the rate of baryon number violating interactions. In our work, however, we also take into account the finite thickness of the wall: this enforces on the parameters of the model some bounds which are stronger than the ones reported in ref. [4]. In section 3 we estimate the thermal corrections to the parameters of the theory, and in particular to the function that measures the suppression of the B violating interactions. Being our model nonrenormalizable, a perturbative treatment can be meaningful only at low energies. Anyhow, it is conceivable that the results we present in section 3 can be a hint for the behavior of the theory at higher temperatures. The issue of baryogenesis is faced in section 4, where we consider a very simple example reminiscent of GUT baryogenesis. In this mechanism, the baryon asymmetry is achieved through the dacay of a boson, whose interactions violate baryon number. In order for the model to work, the boson must be out of equilibrium before decaying, and this is not obvious in theories with low fundamental masses. In those theories, the Hubble parameter is indeed very low at energies below the fundamental scale of gravity, that sets the natural cutoff of the theory. We will thus consider a model where the bosons responsible for the baryon asymmetry are produced nonthermally (for instance, at the end of inflation) fulfilling thus naturally the out of equilibrium requirement. After considering other bounds, such as the stability of the kink under thermal corrections, we finally calculate the baryon asymmetry in a $B-L$ conserving scheme. In the conclusions we discuss our results and their possible future extensions.

\section{LOCALIZATION}

A simple mechanism for localizing fermions on a wall has been recently revisited in ref. [4.

In this paper the idea is illustrated in the easiest case where only one extra dimension is added to the usual four. The main ingredient that is needed is a scalar field $\phi$ which couples to the fermionic field $\psi$ through the full five dimensional Yukawa interaction $g \phi \bar{\psi} \psi$ and whose expectation value $\langle\phi\rangle$ varies along the extra dimension, but it is constant on our four-dimensional world. [

It is possible to show [8] that in this case the fermionic field localizes where its total mass $m=m_{0}+g\langle\phi\rangle\left(m_{0}\right.$ is the bare fermionic mass in the five dimensional theory) vanishes, i.e. on a wall with three spatial dimensions characterized by a particular position $x_{5}$ in the transverse direction.

For definiteness, we consider the theory described by the lagrangian

$$
\begin{aligned}
\mathcal{L}_{\phi \psi} & =\bar{\psi}\left(i \partial_{5}+\frac{1}{\widetilde{M}_{0}^{1 / 2}} \phi(y)+m_{0}\right) \psi \\
\mathcal{L}_{\phi} & =\frac{1}{2} \partial_{\mu} \phi \partial^{\mu} \phi-\left(-\mu_{0}^{2} \phi^{2}+\lambda_{0} \phi^{4}\right),
\end{aligned}
$$

where $y \equiv x_{5}$ is the fifth coordinate, the fields and the parameters have the following mass dimensions

$$
[\phi]=3 / 2,[\psi]=2,\left[m_{0}\right]=\left[\mu_{0}\right]=\left[\widetilde{M}_{0}\right]=1,\left[\lambda_{0}\right]=-1,
$$

\footnotetext{
${ }^{1}$ See also [⿵门日⿹勹巳 for alternative suggestions.

${ }^{2}$ There exist other proposals for baryogenesis in these theories [7,9,10]: in the work [7], after considering several bounds on baryogenesis with large extra dimensions, a mechanism based on nonrenormalizable operators is proposed; in ref. [9] baryon number is violated by "evaporation" of brane bubbles that carry a net baryonic charge into the bulk, and the matter-antimatter asymmetry can be due to a primordial collision of our brane with another one, that carried away the missing antimatter; in ref. [10] baryogenesis is obtained via leptogenesis, the latter being due to the existence of sterile neutrinos in the bulk.

${ }^{3}$ In this way the VEV $\langle\phi\rangle$ breaks the full translational invariance, as it is needed to have a preferred direction orthogonal to the wall.
} 
and where the suffix 0 indicates the value of the parameters at zero temperature.

As we said, the localization position of the fermions depends on the vacuum configuration of the field $\phi$. If we consider the kink solution

$$
\phi=\frac{\mu_{0}}{\sqrt{2 \lambda_{0}}} \tanh \left(\mu_{0} y\right)
$$

and we approximate it with a straight line interpolating between the two vacua (see figure 1)

$$
\left\{\begin{array}{l}
\phi(y) \simeq \frac{\mu_{0}^{2}}{\sqrt{2 \lambda_{0}}} y,|y|<\frac{1}{\mu_{0}} \\
\phi(y) \simeq \pm \frac{\mu_{0}^{2}}{\sqrt{2 \lambda_{0}}}, \quad|y|>\frac{1}{\mu_{0}},
\end{array}\right.
$$

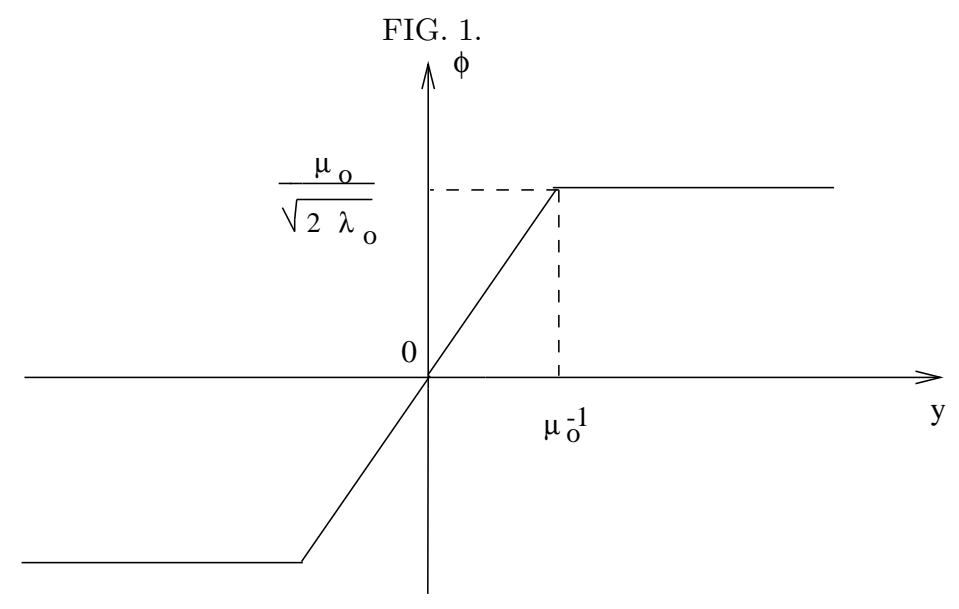

we see that the localization can occur only if

$$
m_{0}<\frac{\mu_{0}}{\sqrt{2 \lambda_{0} \widetilde{M}_{0}}}
$$

since otherwise the total fermion mass

$$
m_{\mathrm{tot}}=\frac{1}{\widetilde{M}_{0}^{1 / 2}} \phi(y)+m_{0}
$$

never vanishes.

It can be shown that, from the four dimensional point of view, a left handed chiral massless fermionic field results from the localization mechanism, if the above configuration (3) is assumed for the scalar $\phi$. The right handed part remains instead delocalized in the whole space. This is not a problem since it is customary to limit the Standard Model and the MSSM fermionic content only to left handed fields. The right handed fields can also be localized if a kink-antikink solution is assumed for the scalar $\phi$. As a result, the left fields continue to be localized on the kink, while the right ones are confined to the antikink. If the kink and the antikink are sufficiently far apart, the left handed and right handed fermions however do not interact and again the model reproducing our four dimensional world must be built by fermions of a defined chirality. The fermionic content of the full dimensional theory is in this case doubled with respect to the usual one, and observers on one of the two walls will refer to the other as to a "mirror world". The presence of this kink-antikink configuration may be required by stability consideration if thermal effects are considered, as it is the case in the next sections. However most of the physics in one brane is not affected by the presence of the mirror one, and in the most of the present work we will concentrate on a single wall as if only the kink (3) configuration was present.

\footnotetext{
${ }^{4}$ Concerning the cancellation of anomalies on the wall and recovering the Standard Model running of the coupling constants, see 11.
} 
In order to give mass to the fermions, some other scalar field acting as a Higgs in the four dimensional theory must be considered. As it is shown in ref. 伆, the mechanism described above could give an explanation to the hierarchy among the Yukawa couplings responsible for the fermionic mass matrix. If indeed one chooses different five dimensional bare masses for the different fermionic fields, the latter are localized at different positions in the fifth direction. As a consequence, the wave functions of different fermions do only partially overlap, and increasing the difference between the five dimensional bare masses of two fermions results in suppressing their mutual interactions.

The same idea can be adopted to guarantee proton stability. Let us give, respectively, leptons and baryons the "masses"

$$
\left(m_{0}\right)_{l}=0, \quad\left(m_{0}\right)_{b}=m_{0},
$$

which correspond to the localizations 5

$$
y_{l}=0 \quad, \quad y_{b}=\frac{m_{0} \sqrt{2 \lambda_{0} \widetilde{M}_{0}}}{\mu_{0}^{2}}<\frac{1}{\mu_{0}}
$$

The shape of the fermion wave functions along the fifth dimension can be cast in an explicit and simple form if we consider the limit $y_{b} \ll 1 / \mu_{0}$, in which the effect of the plateau for $y>1 / \mu_{0}$ can be neglected:

$$
\begin{aligned}
& f_{l}(y)=\left(\frac{\mu_{0}^{2}}{\sqrt{2 \lambda_{0} \widetilde{M}_{0}} \pi}\right)^{1 / 4} \exp \left\{-\frac{\mu_{0}^{2} y^{2}}{2 \sqrt{2 \lambda_{0} \widetilde{M}_{0}}}\right\} \\
& f_{b}(y)=\left(\frac{\mu_{0}^{2}}{\sqrt{2 \lambda_{0} \widetilde{M}_{0}} \pi}\right)^{1 / 4} \exp \left\{-\frac{\mu_{0}^{2}\left(y-y_{b}\right)^{2}}{2 \sqrt{2 \lambda_{0} \widetilde{M}_{0}}}\right\} .
\end{aligned}
$$

We assume the Standard Model to be embedded in some theory which, in general, contains some additional bosons $X$ whose interactions violate baryon number conservation. If it is the case, the four fermion interaction $q q \longleftrightarrow q l$ can be effectively described by

$$
\int d^{4} x d y \frac{q q q l}{\Lambda m_{X}^{2}}
$$

where $m_{X}$ is the mass of the intermediate boson $X$ and $\Lambda$ is a parameter of mass dimension one related to the five-dimensional coupling of the X-particle to quarks and leptons.

This scattering is thus suppressed by

$$
\begin{aligned}
& I=\frac{1}{\Lambda m_{X}^{2}} \int d y \frac{\mu_{0}^{2}}{\pi \sqrt{2 \lambda_{0} \widetilde{M}_{0}}} \exp \left\{-\frac{\mu_{0}^{2} / 2}{\sqrt{2 \lambda_{0} \widetilde{M}_{0}}}\left[y^{2}+3\left(y-y_{b}\right)^{2}\right]\right\}= \\
& \ldots=\frac{\mu_{0}}{\Lambda m_{X}^{2} \sqrt{2 \pi}\left(2 \lambda_{0} \widetilde{M}_{0}\right)^{1 / 4}} \exp \left\{-\frac{3\left(2 \lambda_{0} \widetilde{M}_{0}\right)^{1 / 2}}{8} \frac{m_{0}^{2}}{\mu_{0}^{2}}\right\} .
\end{aligned}
$$

Current proton stability requires $I \lesssim\left(10^{16} \mathrm{GeV}\right)^{-2}$, that is

$$
\frac{m_{0}}{\mu_{0}} \gtrsim \frac{\sqrt{200-6 \log _{10}\left(\frac{\Lambda m_{X}^{2}}{\mu_{0}} / \mathrm{GeV}^{2}\right)}}{\left(2 \lambda_{0} \widetilde{M}_{0}\right)^{1 / 4}} .
$$

\footnotetext{
${ }^{5}$ The last inequality in the next expression comes from (5). We assume quarks of different generations to be located in the same $y$ position in order to avoid dangerous FCNC mediated by the Kaluza-Klein modes of the gluons 12 .

${ }^{6}$ This is also the limit in which the approximation (4) is valid.
} 
The numerator in the last equation is quite insensitive to the mass scales of the model, and - due to the logarithmic mild dependence - can be safely assumed to be of order 10 . For definiteness, we will thus fix it at the value of 10 in the rest of our work.

Conditions (5) and (10) give altogether

$$
\frac{10 \mu_{0}}{\left(2 \lambda_{0} \widetilde{M}_{0}\right)^{1 / 4}} \lesssim m_{0} \lesssim \frac{\mu_{0}}{\left(2 \lambda_{0} \widetilde{M}_{0}\right)^{1 / 2}}
$$

that we can rewrite

$$
\left\{\begin{array}{l}
2 \lambda_{0} \widetilde{M}_{0} \lesssim 10^{-4} \\
\frac{m_{0}}{\mu_{0}} \gtrsim 10^{2}
\end{array}\right.
$$

The last limit in eqs. (12) is stronger than the one given in ref. [4] where proton stability is satisfied if the ratio of the massive scales of the model is of order 10 . However, in ref. [4] the field $\phi$ simply scales linearly as a function of $y$, while we expect that whenever a specific model is assumed, conditions analogous to our (5) and (12) should be imposed.

\section{THERMAL CORRECTION TO THE COEFFICIENTS}

Once the localization mechanism is incorporated in a low energy effective theory - as the system (1) may be considered -, one can legitimately ask if thermal effects could play any significant role. In the present work we are mainly interested in any possible change in the argument of the exponential in eq. (9), that will be the most relevant for the purpose of baryogenesis. For this reason, we introduce the dimensionless quantity

$$
a(T)=\frac{m(T)^{2}}{\mu(T)^{2}} \sqrt{2 \lambda(T) \widetilde{M}(T)}
$$

From eqs.(10) and (12), we can set $a(0) \gtrsim 100$ at zero temperature. Thermal effects will modify this value. There are however some obstacles that one meets in evaluating the finite temperature result. Apart from some technical difficulties arising from the fact that the scalar background is not constant, the main problem is that nonperturbative effects may play a very relevant role at high temperature. As it is customary in theories with extra dimensions, the model (1) is nonrenormalizable and one expects that there is a cut-off (generally related to the fundamental scale of gravity) above which it stops holding. Our considerations will thus be valid only for low temperature effects, and may only be assumed as a rough indication for what can happen at higher temperature.

Being aware of these problems, by looking at the dominant finite-temperature one-loop effects, we estimate the first corrections to the relevant parameters to be

$$
\left\{\begin{array}{l}
\lambda(T)=\lambda_{0}+c_{\lambda} \frac{T}{\widetilde{M}_{0}^{2}} \\
\widetilde{M}(T)=\widetilde{M}_{0}+c_{\widetilde{M}} T \\
m(T)=m_{0}+c_{m} \frac{T^{2}}{\widetilde{M}_{0}} \\
\mu^{2}(T)=\mu_{0}^{2}+c_{\mu} \frac{T^{3}}{\widetilde{M}_{0}}
\end{array}\right.
$$

where the $c$ 's are dimensionless coefficients whose values are related to the exact particle content of the theory.

In writing the above equations, the first of conditions (12) has also been taken into account. For example, both a scalar and a fermionic loop contribute to the thermal correction to the parameter $\lambda_{0}$. While the contribution from the former is of order $\lambda_{0}^{2} T$, the one of the latter is of order $T / \widetilde{M}_{0}^{2}$ and thus dominates. 7

Substituting eqs. (14) into eq. (13), we get, in the limit of low temperature,

\footnotetext{
${ }^{7}$ Notice also that in our model loops with internal leptons dominate over loops with internal quarks, since the former have vanishing five dimensional bare mass and thus are not Boltzmann suppressed. However, although this choice is the simplest one, one may equally consider the most general case where all the fermions have a nonvanishing five dimensional mass.
} 


$$
a(T) \simeq a(0) \cdot\left[1+\frac{T}{\widetilde{M}_{0}}\left(\frac{c_{\lambda}}{2 \lambda_{0} \widetilde{M}_{0}}+\frac{c_{\widetilde{M}}}{2}+\frac{2 c_{m} T}{m_{0}}-\frac{c_{\mu} T^{2}}{\mu_{0}^{2}}\right)\right]
$$

From the smallness of the quantity $\lambda_{0} \widetilde{M}_{0}$ [see cond. (12)] we can safely assume (apart from high hierarchy between the $c$ 's coefficients that we do not expect to hold) that the dominant contribution in the above expression comes from the term proportional to $c_{\lambda}$.

We thus simply have

$$
a(T) \simeq a(0)\left(1+c_{\lambda} \frac{T}{2 \lambda_{0} \widetilde{M}_{0}^{2}}\right) .
$$

We notice that the parameter $c_{\lambda}$, being related to the thermal corrections to the $\phi^{4}$ coefficient due to a fermion loop, is expected to be negative [13]: the first thermal effect is to decrease the value of the parameter $a(T)$, making hence the baryon number violating reactions more efficient at finite rather than at zero temperature.

There is another effect which may be very crucial at finite temperature, linked to the stability of the $Z_{2}$ symmetry. When a temperature is turned on, we generally expect the formation of a fermion-antifermion condensate $\langle\bar{\psi} \psi\rangle \neq 0$. If it is the case, the Yukawa coupling $\phi \bar{\psi} \psi$ in the lagrangian (1) renders one of the two vacua unstable. While this leads to an instantaneous decay of the kink configuration, a kink-antikink system could have a sufficiently long lifetime provided the two objects are enough far apart.

\section{BARYOGENESIS}

We saw in the previous section that thermal effects may increase the rate of baryon number violating interactions of our system. This is very welcome, since a theory which never violates baryon number cannot lead to baryogenesis and thus cannot reproduce the observed Universe. Anyhow baryon number violation is only one of the ingredients for baryogenesis, and the aim of this section is to investigate how the above mechanism can be embedded in a more general context.

A particular scheme which may be adopted is baryogenesis through the decay of massive bosons $X$. 1 This scheme closely resembles GUT baryogenesis, but there are some important peculiarities due to the different scales of energy involved. In GUT baryogenesis the massive boson $X$, coupled to matter by the interaction $g X \psi \bar{\psi}$, has the decay rate

$$
\Gamma \simeq \alpha m_{x}, \quad \alpha=\frac{g^{2}}{4 \pi} .
$$

An important condition is that the $X$ boson decays when the temperature of the Universe is below its mass (out of equilibrium decay), in order to avoid thermal regeneration. From the standard equation for the expansion of the Universe,

$$
H \simeq g_{*}^{1 / 2} \frac{T^{2}}{M_{p}}
$$

(where $g_{*}$ is the number of relativistic degrees of freedom at the temperature $T$ ), this condition rewrites

$$
m_{X} \gtrsim g_{*}^{-1 / 2} \alpha M_{p} .
$$

If $X$ is a Higgs particle, $\alpha$ can be as low as $10^{-6}$. Even in this case however the $X$ boson must be very massive. In principle this may be problematic in the theories with extra dimensions we are interested in, which have the main goal of having a very low fundamental scale.

There are some possibilities to overcome this problem. One is related to a possible deviation of the expansion of the Universe from the standard behavior. The issue of the Friedmann law in models with large extra dimensions has been indeed subject of intense debate in the recent past. In the work 14 a detailed analysis of the Einstein equations with

\footnotetext{
${ }^{8}$ We may think of these bosons as the intermediate particles which mediate the four fermion interaction described by the term (8).
} 
one extra dimension, shows that the expansion rate $H$ should (in absence of any energy in the bulk) be proportional to the energy density $\rho$ on the brane. This behavior strongly conflicts with the standard one $H \propto \rho^{1 / 2}$. In refs. [15-17] it was then shown that the standard expansion law could be achieved, at least at low temperatures, by a suitable fine tuning of the vacuum energies in the Universe. In particular, this is the case for the Randall-Sundrum model [2], which offers one of the most satisfactory solutions to the hierarchy problem. The solution proposed in these works 15 17] is however itself plagued by some other cosmological problems. For example, in the RS model [2] gravity turns out to be repulsive on our brane. All the above problems are overcome when some mechanism for the stabilization of the radion is taken into account, as the analyses [18,19] show. In particular, in ref. [18], the case of the model [2] is examined, and both the standard Friedmann law and the "correct" sign for the Newton constant are obtained. The analysis of ref. [18] is however performed by computing only the first-order term of the expansion of the square of the Hubble parameter $H^{2}$ as a power series of the energy density $\rho$. Terms of order $\rho^{2}$ could become relevant at temperatures above $1 \mathrm{TeV}$ or so [18]. This may result in an accelerated expansion of the Universe at high temperatures, and the out of equilibrium condition for the $X$ bosons could be consequently considerabily favoured.

However, both the facts that we do not know the exact behavior of the expansion rate at high temperatures in the model [2], and that one may be interested in embedding our baryogenesis mechanism in some other cosmological scenario, lead us to discuss alternative solutions for the out of equilibrium problem. One very natural possibility is to create the $X$ particles non thermally and to require the temperature of the Universe to be always smaller than their mass $m_{X}$. In this way, one kinematically forbids regeneration of the $X$ particles after their decay. In addition, although interactions among these bosons can bring them to thermal equilibrium, chemical equilibrium cannot be achieved.

Nonthermal creation of matter has raised a considerable interest in the last years. In particular, it has been shown that this production can be very efficient during the period of coherent oscillations of the inflaton field after inflation [20 22]. The efficiency of this mechanism has also been exploited in the work [23] to revive GUT baryogenesis in the context of standard four dimensional theories. Here, we will not go into the details of the processes that could have lead to the production of the $X$ bosons. Rather, we will simply assume that, after inflation, their number density is $n_{X}$. To simplify our computations, we will also suppose that their energy density dominates over the thermal bath produced by the perturbative decay of the inflaton field.

Just for definiteness, let us consider a very simple model where there are two species of $X$ boson which can decay into quarks and leptons, according to the four dimensional effective interactions

$$
g X \bar{q} \bar{q}, \quad g e^{-a / 4} X l q,
$$

where (remember the suppression given by the different localization of quarks and leptons) the quantity $a$ is defined in eq. (13). Again for definiteness we will consider the minimal model where no extra fermionic degrees of freedom are added to the ones present in the Standard Model. Moreover we will assume $B-L$ to be conserved, even though the extension to a more general scheme can be easily performed.

The decay of the $X$ bosons will reheat the Universe to a temperature that can be evaluated to be

$$
T_{\mathrm{rh}} \simeq\left(\frac{30}{\pi^{2}} \frac{m_{X} n_{X}}{g_{*}}\right)^{1 / 4} .
$$

Since we do not want the $X$ particles to be thermally regenerated after their decay, we require $T_{\text {rh }} \lesssim m_{X}$, that can be rewritten as an upper bound on $n_{X}$

$$
n_{X} \lesssim 30\left(\frac{g_{*}}{100}\right) m_{X}^{3} .
$$

Another limit comes from the necessity to forbid the $B$ violating four fermion interaction (8) to erase the $B$ asymmetry that has been just created by the decay of the $X$ bosons. We thus require the interaction (8) to be out equilibrium at temperatures lower than $T_{\mathrm{rh}}$. From eq. (9) we see that we can parametrize the four fermion interaction with a coupling $g^{2} e^{-3 a / 8} / m_{X}^{2}$. Hence, the out of equilibrium condition reads

$$
g^{4} e^{-3 a / 4} \lesssim g_{*} \frac{m_{X}}{M_{p}}\left(\frac{m_{X}}{T_{\mathrm{rh}}}\right)^{3} .
$$

One more upper bound on the reheating temperature comes from the out of equilibrium condition for the sphalerons. This requirement is necessary only if one chooses the theory to be $B-L$ invariant, while it does not hold for $B-L$ violating schemes. We can approximately consider the sphalerons to be in thermal equilibrium at temperatures above the electroweak scale. Thus, if $B-L$ is a conserved quantity, we will require the reheat temperature to be smaller than about $100 \mathrm{GeV}$. 
If one neglects the presence of the thermal bath prior to the decay of the $X$ bosons, the very first decays will be only into couples of quarks, since the channel into one quark and one lepton is strongly suppressed by the $e^{-a(T=0)}$ factor due to the fact that the kink is not modified by any thermal correction. However, the decay process is not an instantaneous event. It is shown in ref. 24 that the particles produced in the very first decays are generally expected to thermalize very rapidly, so to create a thermal bath even when most of the energy density is still stored in the decaying particles. 9 The temperature of this bath can even be considerably higher than the final reheating themperature. The presence of the heat bath modifies in turn the shape of the kink, as shown in the previuos section, and we can naturally expect that this modification enhances the $B$ violating interaction.

If the energy density of the Universe is dominated by the $X$ bosons before they decay, one has

$$
\eta_{B} \simeq 0.1\left(N_{X} T_{\mathrm{rh}} / m_{X}\right)\langle r-\bar{r}\rangle
$$

where $N_{X}$ is the number of degrees of freedom associated to the $X$ particles and $\langle r-\bar{r}\rangle$ is the difference between the rates of the decays $X \rightarrow q l$ and $\bar{X} \rightarrow \bar{q} \bar{l}$.

We denote with $X_{1}$ and $X_{2}$ the two species of bosons whose interactions (20) lead to baryon number violation, and parametrize by $\epsilon$ the strength of CP-violation in these interactions. Considering that $e^{-2 a}$ is always much smaller than one, we get 25.

$$
\langle r-\bar{r}\rangle \sim 3 g^{2} e^{-a / 2} \epsilon \operatorname{Im} I_{S S}\left(M_{X_{1}} / M_{X_{2}}\right)
$$

where the function $\operatorname{Im} \operatorname{I}_{S S}(\rho)=\left[\rho^{2} \log \left(1+1 / \rho^{2}\right)-1\right] /(16 \pi)$ can be estimated to be of order $10^{-3}-10^{-2}$. It is also reasonable to assume $\epsilon \sim 10^{-2}-1$.

Collecting all the above estimates, and assuming $N_{X}$ to be of order 10, we get

$$
\eta_{B} \simeq\left(10^{-5}-10^{-2}\right) g^{2} \frac{T_{\mathrm{rh}}}{m_{X}} e^{-a\left(T_{\mathrm{rh}}\right) / 2} .
$$

From the requirement $T_{\mathrm{rh}} \lesssim m_{X}$ we get an upper limit on the baryon asymmetry

$$
\eta_{B} \lesssim\left(10^{-5}-10^{-2}\right) g^{2} e^{-a / 2}
$$

where the factor $a(T)$ has to be calculated for a value of $T$ of the order of the reheating temperature.

We get a different limit on $\eta_{B}$ from the bound (23): assuming $m_{X} \sim \mathrm{TeV}$ and $g_{*} \sim 100$ indeed one obtains

$$
\eta_{B} \lesssim\left(10^{-6}-10^{-10}\right) g^{2 / 3} e^{-a / 4}
$$

Since the observed amount of baryon asymmetry is of order $10^{-10}$, even in the case of maximum efficiency of the process (that is, assuming maximal $C P$ violation and $g \sim 1$ ), we have that both bounds (27) and (28) imply that $a\left(T_{\mathrm{rh}}\right)$ has to be smaller than about 40 .

Unfortunately, the temperature at which the condition $a(T) \lesssim 40$ occurs cannot be evaluated by means of the expansion of eq. (16), that have been obtained under the assumption $|a(T)-a(0)| \ll a(0)$. On the other hand, it is remarkable that our mechanism may work with a ratio $a\left(T_{d}\right) / a(0)$ of order one. We thus expect that a successful baryogenesis may be realized for a range of the parameters of our theory which - although not evaluable through a perturbative analysis - should be quite wide and reasonable.

In scenarios with large extra dimensions and low scale gravity, the maximal temperature reached by the Universe after inflation is strongly bounded from above in order to avoid overproducing Kaluza-Klein graviton modes, which may eventually contradict cosmological observations [26]. For instance, in models with two large extra dimensions the reheating temperature has to be less than about $10 \mathrm{MeV}$. This value would be too low for our scenario since $\eta_{B}$ is proportional to the ratio $T_{\mathrm{rh}} / m_{X}$, and hence the observed amount of baryons would be reproduced at the price of an unnaturally small value of $a\left(T_{\mathrm{rh}}\right)$. However, other schemes with extra dimensions exist where the bounds on $T_{\mathrm{rh}}$ are less severe. For example, in the proposals 2,27] the mass of the first graviton KK mode is expected to be of order $\mathrm{TeV}$. The reheating temperature can thus safely be taken to be of order $10-100 \mathrm{GeV}$.

An alternative way to overcome the bound (19) relies on the fact that, as observed in the work [24], the maximal temperature reached by the thermal bath during reheating can indeed be much higher than the final reheating

\footnotetext{
${ }^{9}$ As shown in ref. 24], what is called the reheating temperature is indeed the temperature of the thermal bath when it starts to dominate. After the first decays, the temperature of the light degrees of freedom can be even much higher than $T_{\text {rh }}$.
} 
temperature. In this case, even if $T_{\mathrm{rh}}$ is considerably lower than $m_{X}, X$ particles can be produced in a significant amount, and the out of equilibrium condition is easily achieved. However, the treatment of this mechanism is in our case somewhat different from the one given in ref. 24]: due to the slowness of the expansion of the Universe, the $X$ bosons will decay before the freeze out of their production. The final baryon asymmetry cannot be estimated with the use of the formulae of [24], which are valid only if the decay of the $X$ particles occurs well after their freeze out.

There are of course several possible baryogenesis schemes alternative to the one just presented. A possible option which also requires a minimal extension to the Standard Model could be to achieve the baryon asymmetry directly through the 4 fermions interactions $q+q \leftrightarrow q+l$ in the thermal primordial bath. The out of equilibrium condition may be provided by the change of the kink as the temperature of the bath decreases. 10 What may be problematic is the source of $C P$ violation which may lead the creation of the baryon asymmetry. A possibility in this regard may be provided by considering a second Higgs doublet, but the whole mechanism certainly deserves a deep analysis on its own.

\section{CONCLUSIONS}

The present work concerns the important issue of baryogenesis in theories with large extra-dimensions. Since the observed proton stability requires to a very high degree of accuracy baryon conservation at zero temperature, this task may be problematic within the above theories, which have very low fundamental scales.

Our proposal relies on the localization mechanism for fermions discussed in ref. [4]. While in this work the present proton stability is due to a different localization (in the transverse direction) of leptons and quarks, we believe that thermal corrections may activate early baryon violating interactions.

In our work we first provide a general discussion of the above scheme, without referring to any particular mechanism of baryogenesis. We find indeed that the first thermal corrections are in the direction of increasing the rate of baryon violations.

We then consider a very specific example, where the matter-antimatter asymmetry is achieved through the decay of a (relatively) heavy boson in a $B-L$ conserving context. In this situation the Sakharov out of equilibrium condition can be obtained in the simplest way by considering nonthermal production of the bosons responsible for $B+L$ violation.

Several bounds apply to the whole mechanism. The most general ones concern the localization procedure (we have found that the limits given in ref. [4 become more stringent once the thickness of the wall is considered) and its stability against thermal corrections. In addition, there are some other constraints which hold in the particular scheme of baryogenesis we adopted. The temperature of the heat bath right after the production of the baryon asymmetry cannot be too high, to avoid thermal regeneration of the bosons that induced baryogenesis. Moreover, this temperature has not to exceed the electroweak scale, in order not to activate the sphaleron transitions that would erase the $B+L$ asymmetry produced at some higher energy. Of course, this last bound can be easily overcome by considering some $B-L$ nonconserving process.

We have found that the observed baryonic asymmetry can be accomplished quite naturally in our example, and we believe this should be the case in a more general context as well.

Possible extensions of the present work are related to the generality of the scenario we discussed. Our idea indeed relies only on the localization mechanism adopted in [4 and not on the geometry of the bulk, nor on the details of the interactions responsible for the baryon asymmetry.

Future works could thus proceed in two directions. Firstly, one could try to embed the scheme here described in a more complete cosmological setting. Secondly, some other baryogenesis mechanisms, for example à la Affleck-Dine (which does not require very high energy scales), may be explored.

\section{ACKNOWLEDGMENTS}

We thank J. March-Russell, G. Mussardo, H.P. Nilles, M. Pietroni, and A. Riotto for interesting and stimulating discussions. This work is partially supported by the MURST research project "Astroparticle Physics".

\footnotetext{
${ }^{10}$ This condition may be easily achieved due of the exponential dependence of the rate of this process on the temperature, see eq. (9).
} 
[1] N. Arkani-Hamed, S. Dimopoulos and G. Dvali, Phys. Lett. B429 (1998) 263 hep-ph/9803315.

[2] L. Randall and R. Sundrum, Phys. Rev. Lett. 83 (1999) 3370 [hep-ph/9905221].

[3] L. Randall and R. Sundrum, Phys. Rev. Lett. 83 (1999) 4690 [hep-th/9906064].

[4] N. Arkani-Hamed and M. Schmaltz, Phys. Rev. D61 (2000) 033005 hep-ph/9903417.

[5] I. Antoniadis, N. Arkani-Hamed, S. Dimopoulos and G. Dvali, Phys. Lett. B436 (1998) 257 hep-ph/9804398.

[6] G. Shiu and S. H. Tye, Phys. Rev. D58 (1998) 106007 hep-th/9805157.

[7] K. Benakli and S. Davidson, Phys. Rev. D60 (1999) 025004 [hep-ph/9810280].

[8] V. A. Rubakov and M. E. Shaposhnikov, Phys. Lett. B125 (1983) 136.

[9] G. Dvali and G. Gabadadze, Phys. Lett. B460 (1999) 47 hep-ph/9904221.

[10] A. Pilaftsis, Phys. Rev. D60 (1999) 105023 hep-ph/9906265].

[11] S. Randjbar-Daemi and J. Strathdee, Nucl. Phys. B461 (1996) 305 hep-th/9510067.

[12] A. Delgado, A. Pomarol and M. Quiros, JHEP 0001 (2000) 030 hep-ph/9911252.

[13] K. R. Dienes, E. Dudas, T. Gherghetta and A. Riotto, Nucl. Phys. B543 (1999) 387 [hep-ph/9809406.

[14] P. Binetruy, C. Deffayet and D. Langlois, hep-th/9905012.

[15] C. Csaki, M. Graesser, C. Kolda and J. Terning, Phys. Lett. B462, 34 (1999) hep-ph/9906513.

[16] J. M. Cline, C. Grojean and G. Servant, Phys. Rev. Lett. 83 (1999) 4245 hhep-ph/9906523.

[17] T. Shiromizu, K. Maeda and M. Sasaki, gr-qc/9910076.

[18] C. Csaki, M. Graesser, L. Randall and J. Terning, hep-ph/9911406.

[19] P. Kanti, I. I. Kogan, K. A. Olive and M. Pospelov, hep-ph/9912266.

[20] J. H. Traschen and R. H. Brandenberger, Phys. Rev. D42 (1990) 2491.

[21] L. Kofman, A. Linde and A. A. Starobinsky, Phys. Rev. Lett. 73 (1994) 3195 hep-th/9405187.

[22] Y. Shtanov, J. Traschen and R. Brandenberger, Phys. Rev. D51 (1995) 5438 hep-ph/9407247.

[23] E. W. Kolb, A. Linde and A. Riotto, Phys. Rev. Lett. 77 (1996) 4290 hep-ph/9606260].

[24] D. J. Chung, E. W. Kolb and A. Riotto, Phys. Rev. D60 (1999) 063504 hep-ph/9809453].

[25] D. V. Nanopoulos and S. Weinberg, Phys. Rev. D20 (1979) 2484.

[26] N. Arkani-Hamed, S. Dimopoulos and G. Dvali, Phys. Rev. D59 (1999) 086004 hep-ph/9807344.

[27] N. Kaloper, J. March-Russell, G. D. Starkman and M. Trodden, hep-ph/0002001. 